\section{SCIENCE CHINA \\ Physics, Mechanics \& Astronomy}

-Editorial•

Editor's Focus
October 2021 Vol. 64 No. 10: 107461

https://doi.org/10.1007/s11433-021-1763-4

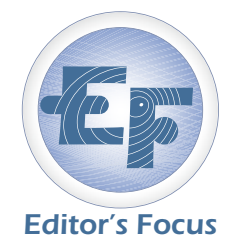

\title{
Nodeless superconductivity in a vanadium kagome metal
}

The pairing symmetry of the superconducting order parameter contains key information about the pairing mechanism of the superconductor. For conventional superconductors, whose pairing is usually induced by the electron-phonon interaction, the pairing symmetry is s-wave, and the superconducting gap is fully open everywhere on the Fermi surface. For unconventional superconductors such as the cuprate and heavy fermion superconductors, other pairing symmetries may occur, leading to symmetry-mandated nodes in the superconducting order parameter. For any newly discovered superconductor, it is essential to know its pairing symmetry and the corresponding pairing mechanism. For most unconventional superconductors, determining the pairing mechanism remains a challenging problem.

Recently, a family of V-based kagome superconductors has come to the fore [1,2], drawing concerted research efforts from the condensed matter physics community. The interest in these materials lies in their charge density wave order, which may realize the long-sought time-reversal-symmetry-breaking by loop-current [3], and a superconducting ground state that emerges in its presence on a nearly perfect kagome lattice [2]. While the understanding of the pairing symmetry and pairing mechanism of this family of superconductors is fluid, a recent study by Duan et al. [4] provides key information about the pairing symmetry, setting limits on the possible pairing mechanisms. In this work, through careful measurements of the magnetic penetration depth and specific heat at low temperatures, the authors show that the superconducting order parameter is fully-gapped, although multiple gaps are required to fully account for the experimental data. These findings rule out many unconventional pairing symmetries, and the corresponding pairing mechanisms.

This work provides a key piece of experimental evidence vital for understanding the newly discovered vanadium kagome superconductors. On the other hand, much remains to be understood about these fascinating materials, including the nature of the charge-density-wave, and whether topological superconductivity can be realized.

Hai-Qing Lin

Beijing Computational Science Research Center, Beijing 100193, China

1 B. R. Ortiz, L. C. Gomes, J. R. Morey, M. Winiarski, M. Bordelon, J. S. Mangum, I. W. H. Oswald, J. A. Rodriguez-Rivera, J. R. Neilson, S. D. Wilson, E. Ertekin, T. M. McQueen, and E. S. Toberer, Phys. Rev. Mater. 3, 094407 (2019).

2 B. R. Ortiz, S. M. L. Teicher, Y. Hu, J. L. Zuo, P. M. Sarte, E. C. Schueller, A. M. M. Abeykoon, M. J. Krogstad, S. Rosenkranz, R. Osborn, R. Seshadri, L. Balents, J. He, and S. D. Wilson, Phys. Rev. Lett. 125, 247002 (2020), arXiv: 2011.06745.

3 Y. X. Jiang, J. X. Yin, M. M. Denner, N. Shumiya, B. R. Ortiz, G. Xu,
Z. Guguchia, J. He, M. S. Hossain, X. Liu, J. Ruff, L. Kautzsch, S. S. Zhang, G. Chang, I. Belopolski, Q. Zhang, T. A. Cochran, D. Multer, M. Litskevich, Z. J. Cheng, X. P. Yang, Z. Wang, R. Thomale, T. Neupert, S. D. Wilson, and M. Z. Hasan, Nat. Mater., https://doi.org/ 10.1038/s41563-021-01034-y.

4 W. Duan, Z. Nie, S. Luo, F. Yu, B. R. Ortiz, L. Yin, H. Su, F. Du, A. Wang, Y. Chen, X. Lu, J. Ying, S. D. Wilson, X. Chen, Y. Song, and H. Yuan, Sci. China-Phys. Mech. Astron. 64, 107462 (2021). 